\title{
INVESTIGATION OF ELECTROHYDRAULIC DRIVE CONTROL SYSTEM WITH THE HAPTIC JOYSTICK
}

\author{
Paweł BACHMAN*, Andrzej MILECKI ** \\ ${ }^{\star}$ Faculty of Mechanical Engineering, University of Zielona Góra, ul. Licealna 9, 65-417 Zielona Góra, Poland \\ ${ }^{* *}$ Faculty of Mechanical Engineering and Management, Poznan University of Technology, ul. Piotrowo 3, 60-965 Poznań, Poland \\ p.bachman@iiibnp.uz.zgora.pl, andrzej.milecki@put.poznan.pl
}

received 10 October 2016, revised 23 February 2018, accepted 26 February 2018

\begin{abstract}
The term haptic is used to indicate the presence of force feedback from the manipulated object to the operator. One of the most commonly used haptic devices are joysticks. Such joysticks can be successfully applied also in communication with drive system, giving the human operator a feel of the output force. In the paper one axis joystick with force feedback used to control the electrohydraulic drive is proposed. In this joystick, a controlled brake with magnetorheological fluid and a small DC motor are applied. A beam with a strain gauge is used in a joystick arm, enabling the measurement of the force. In the joystick axis also a potentiometer is assembled, which measured the current arm position. In order to develop the control algorithms an electrohydraulic drive simulation and virtual model is worked out and then a haptic joystick is connected to it. The simulation results that have been obtained, enabled to design and test impedance and admittance control strategies for the system composed of haptic joystick and a real electrohydraulic drive. Finally the whole system is built, implemented and investigated in a laboratory environment. Investigations are conducted in conditions similar to real ones, in a situation where hydraulic piston touches an obstacle and the operator cannot observe this piston very accurately. Fifteen operators have been tested this way. The outcomes indicate that haptic control can improve the human feeling of forces between electrohydraulic drive and an obstacle and, thanks to this, the manual control is more accurate and safer.
\end{abstract}

Key words: Electrohydraulics, Haptic, Joystick, Control, Modelling

\section{INTRODUCTION}

Different devices like cranes, lifts, excavators are often controlled directly by humans, which use special levers or joysticks to assign the drive position or force. In most of these applications visual and sometimes also sound signals are used by the operator as a feedback. However, for most precise tasks it would be advantageous, to have additional feedback like feeling of the force, produced by the controlled drive (Li, 2000). Having it, the operator would be able to control the system more accurately.

The term haptic is used to indicate the presence of force feedback from the manipulated object to the operator. The aim of haptic interfaces is to render the mechanical features of the object on the user. Haptic solutions can be very valuable, because they can help the human to perform tasks more effectively and more reliably (Harward, 2006). All elements the person would touch using a drive controlled by a joystick, transfer tactile impressions to a joystick and to a user. As haptic devices such elements like: joysticks, steering-wheels, mouse's, pedals etc., are most commonly used. There can also be haptic displays, gloves, dresses and different imitations of real objects like medical instruments or bow from fiddle (Adams and Hannaford, 1999).

Haptic devices have been designed and first used about 40 years ago. Since that time their different solutions and applications are described. Some of them are focused on control of different working machines in which electrohydraulic drives are used. In the article (Zarei-nia et al., 2009) the electrohydraulic drive with proportional valve and force feedback controlled by SensAble Phantom Omni haptic device is described. The investigations are carried out for two types of load and several concepts of control algorithms. The same haptic device used for a control of an excavator arm with electrohydraulic drives is described in thesis (Frankel, 2004). Analog sensors are used to measure the position of the excavator boom and the pressure in the chambers of each cylinder that is used as a feedback signal in the controller. The important part of this work, is focused on the modeling of the hydraulic excavator. The paper (Won Oh et al., 2011) described the simulation tests of a virtual excavator arm in which a special touch device is used. Authors described the simulation results when the virtual excavator arm driven by the hydraulic device affected the wall. In the paper (Park et al., 2011), a similar study for the detection of the collisions occurring in contact between the excavator bucket and environment, is presented. The information from the excavator is transmitted to the operator which used a haptic joystick. It is shown that in a traditional remote control of excavators, in which the image is obtained by a camera and presented to the user on $2 \mathrm{D}$ screen, the movement or the force cannot be properly assessed. This concerns especially the depth of the trench. As a solution, the dual axis haptic joystick with a small motor is proposed and successfully applied and implemented. Paper (Kontz, 2007) contains the description of the excavator control system, in which only the force acting on a bucket is measured. The operator used the haptic device type SensAble Phantom Omni. The investigations have shown that the proposed solution greatly increased the abilities of the operator, giving him additional feedback, which enabled the improvement of the excavator movements coordination. In thesis (Kontz, 2002) 
authors presented a concept of hydraulic forklift controlled remotely by the haptic device type SensAble Phantom Desktop, which is connected to the control system by LAN and TCP/IP protocol. In the fork a sensor is mounted, which measured the force in vertical axis of the movement. The voltage signal from this sensor is used to prepare the force feedback signal. In the article (Zee, 2009) another excavators with hydraulic drives, controlled by a haptic joystick are described. Author has proposed his own design of haptic joystick using DC motors and showing the relationship between the swing of excavators arm and the joystick. In the article (Oh K. W. et al., 2008) another design of haptic joystick used to control the excavator arm is shown. In the paper (Kudomi S. et al., 2000) the concept of the master-slave haptic configuration using two hydraulic cylinders working in the impedance control system is presented. The first hydraulic drive (slave) has moved a load and the second has controlled the position of the demand device (master). The voltage signals for actuators valves are generated by the controller, which used the signals from the force sensors.

\section{MODEL OF HYDRAULIC DRIVE}

Electrohydraulic servo drives are used in applications requiring high forces. The basic hydraulic part consists of the hydraulic cylinder and the hydraulic amplifier, as shown in Fig. 1. The amplifier spool is moved by the electromechanical transducer, which in most cases is a proportional electromagnet.

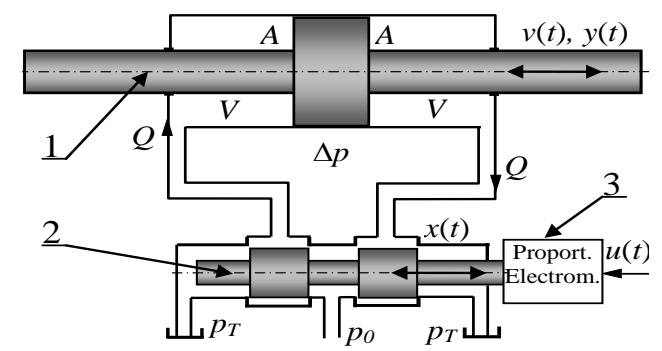

Fig. 1. Hydraulic drive: 1 - hydraulic cylinder, 2 - hydraulic valve, 3 -proportional electromagnet

From the automation point of view, the electrohydraulic drives are non-linear systems, which in many theoretical analyses are linearized. The set of linear equations describing the hydraulic part of the servo drive can be written as follows:
$Q(t)=Q_{s}(t)+Q_{h}(t)+Q_{v}(t)$

where: $Q$ - valve flow, proportional (inlet and outlet), $Q_{s}-$ flow balancing the flow compressibility, $Q_{v}$ - cylinder absorptivity, proportional to the velocity, $Q_{l}-$ leakage flow.

Substituting appropriate linear or linearized equations one obtains (Milecki and Myszkowski, 2003)

$K_{Q} x(t)-K_{p} \Delta p(t)=\frac{v}{2 E_{0}} \frac{d \Delta p(t)}{d t}+A \frac{d y(t)}{d t}+K_{l} \Delta p(t)$

where: $x$ - spool displacement, $y$ - piston position [m], $K_{Q}$ - valve flow gain $\left[\mathrm{m}^{2} / \mathrm{s}\right], K_{p}-$ valve flow-pressure coefficient $\left[\mathrm{m}^{5} /(\mathrm{N} \cdot \mathrm{s})\right]$, $\Delta p$ - pressure difference in a cylinder [Pa], $E_{0}$ - fluid bulk modulus [Pa], $A$ - cylinder piston cross-sectional area $\left[\mathrm{m}^{2}\right], K_{l}$ - leakage coefficient $\left[\mathrm{m}^{5} /(\mathrm{N} \cdot \mathrm{s})\right], V$ - average contained volume of each cylinder chamber in a middle position of the piston [ $\left.\mathrm{m}^{3}\right]$.

The motion of the system can be described as follows

$m \frac{d^{2} y(t)}{d t}+D \frac{d y(t)}{d t}+F_{L}(t)=A \Delta p(t)$

where: $F_{\mathrm{L}}$ - load force [N], $m$ - mass $[\mathrm{kg}], D$ - viscous damping coefficient (Newtonian friction) [Ns/m].

The control valve can be described as second order system described by equation

$\frac{d^{2} x(t)}{d t}=k_{Z} \omega_{Z}^{2} u(t)-2 \zeta_{Z} \omega_{z} \frac{d x(t)}{d t}-\omega_{Z}^{2} x(t)$

where: $k_{z}$ - the valve gain $[\mathrm{m} / \mathrm{V}], \omega_{z}$ - valve natural frequency $[\mathrm{Hz}], \zeta_{z}-$ valve damping ratio, $u$ - electromagnet input signal [V].

Basing on equations $(1) \div(4)$, the electrohydraulic servo drive is described as fifth order system, consisting of valve described by second order system and cylinder described by second order element (usually oscillatory) connected serially to the integrative element. Assuming that the fluid leakage in a cylinder can be omitted, the block scheme of the drive is built and shown in Fig. 2. (Milecki and Myszkowski, 2003). Drive parameters are calculated basing on data characterizing the drive i.e.: cylinder dimensions, load, friction coefficient, oil bulk modulus etc. The valve parameters are taken from catalogue where valve step responses, frequency characteristics etc. are given. In the described here model following parameters are used:

- piston cross section area: $A=0.008 \mathrm{~m}^{2}$,

- flow coefficient: $K_{Q}=1.0 \mathrm{~m}^{2} / \mathrm{s}$,

- valve flow-pressure coefficient: $K_{p}=10^{-9} \mathrm{~m}^{5} / \mathrm{Ns}$,

- cylinder stiffness: $2 E_{0} / V=8 \cdot 10^{12} \mathrm{~Pa} / \mathrm{m}^{3}$,

- dynamic friction coefficient: $D=1000 \mathrm{Ns} / \mathrm{m}$,

- mass: $m=200 \mathrm{~kg}$.

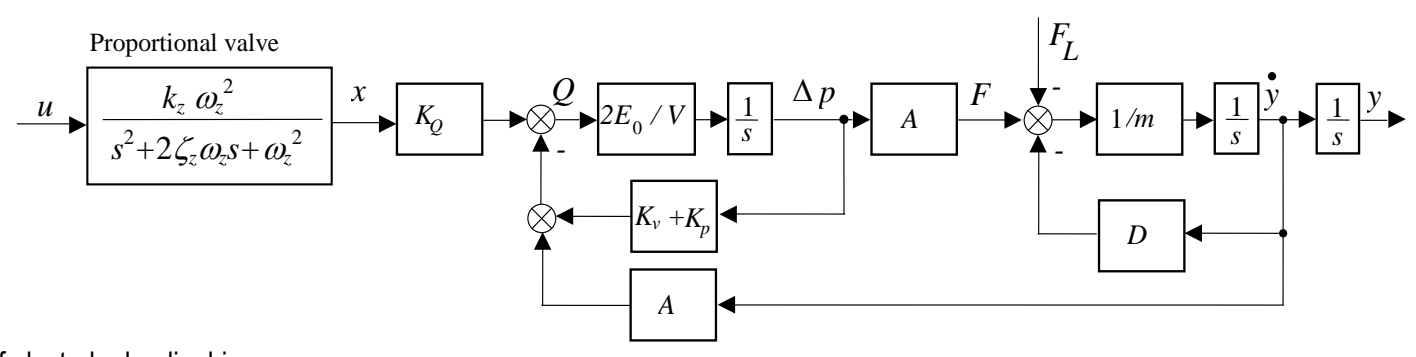

Fig. 2. Model of electrohydraulic drive

Proportional valve is described as a second order system with damping coefficient $\zeta_{z}=1.2$ and with natural frequency $f_{z}=16 \mathrm{~Hz}$. The presented above model should be extended by adding valve control card model. Because the electronic control unit is usually very fast in comparison to the hydraulic part, at first it can be described only by a proportional element. 


\section{HAPTIC JOYSTICK AND ITS APPLICATION IN SIMULATION INVESTIGATION}

In Fig. 3 the view of a one axis joystick prototype and his electronic circuits is shown. In this joystick, in order to generate the movement opposite force the rotary brake with magnetorheological (MR) fluid is used. On the brake axle, the joystick arm in the form of strain gauge load cell is mounted, which serves to measure the forces occurring on the joystick handle. On the other side of the MR brake, a potentiometer is used for measuring of the angular position. On the same axle the gear wheel with a belt is assembled to connect a DC motor.

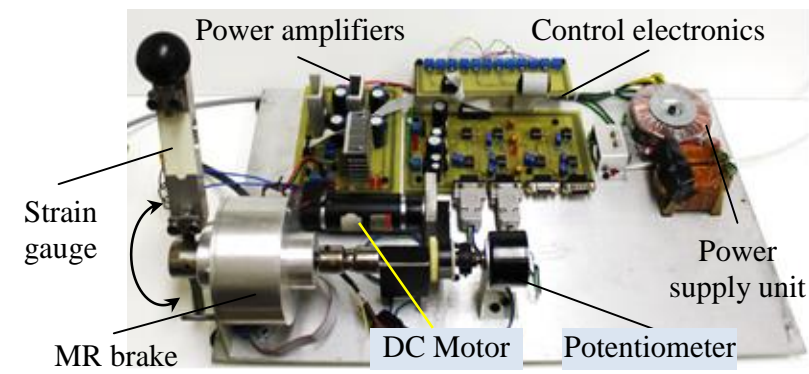

Fig. 3. The photo of the haptic joystick and its controller

The control electronic circuits include: operational amplifiers used to calibrate the joystick position and the force signals; two operational power amplifiers: one for the brake supplying and one for the motor control.

The made in Matlab-Simulink software simulation model of the described above hydraulic drive system with haptic joystick is shown in Fig. 4. It is assumed that the MR brake is modeled by the first order transfer function (with the time constant $0.01 \mathrm{sec}$.) connected serially with time delay equal to $0.05 \mathrm{sec}$. The human operator is modeled as a first order system with time constant equal to $0.1 \mathrm{sec}$. It is also assumed that the human operator control algorithm is PID like. There are two feedback loops in the model: force and position feedback. The first one is made by a haptic joystick with MR brake and the second one is made by an external cylinder piston position loop. The current position is assessed by operator's observation. The model is then connected to a virtual world thanks to special Simulink elements called VR Signal Expander and VR Sink. The first element converts simulation signal into a signal compatible with a VR Modeling Language. In case of simulation described in this article, a servo-cylinder piston displacement signal $y$ is used as the VR movement simulation signal. VR Sink software includes graphic interface, combining simulation results with pictures presented in virtual reality. The Matlab VR environment includes library of a different 3D visualization objects. However, there are no hydraulic elements in this library and therefore authors have built one basing on a real servo-cylinder design principle. This simulation model was used for initial testing of the control of electrohydraulic drive.

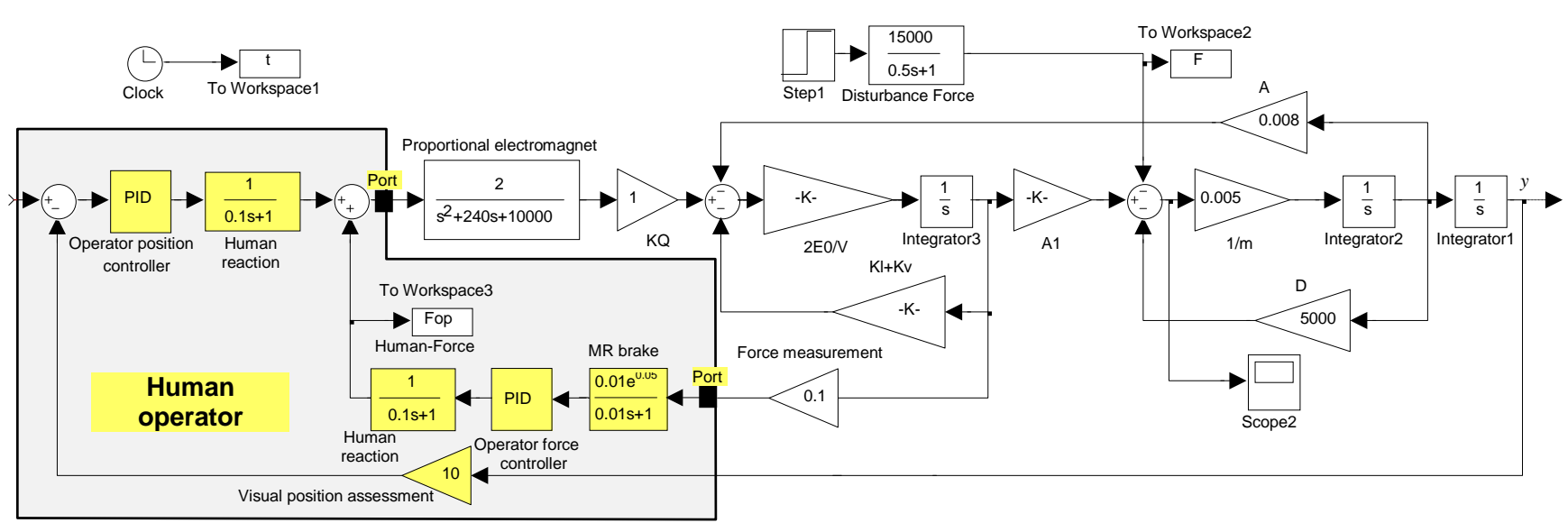

Fig. 4. Model of electrohydraulic drive

In the next step the block "Human operator" is removed from the model and replaced with the real haptic joystick, that is connected to the computer using an input/output card type RT-DAC as shown in Fig. 5. This card is controlled by toolbox software RTCON, which is installed and used to connect the VR model of electrohydraulic drive (EHD) with the haptic joystick. Joystick input signals are: supply voltages of MR brake coil amplifier $\left(U_{M R B}\right)$ and of DC motor $\left(U_{M}\right)$. VR model output signals are: joystick arm angular position $x_{j}$ measured by the potentiometer and joystick force signal $F_{j}$ measured by strain gauge. In the presented system position control feedback loop is closed by the operator's eyesight (dashed line in the Fig. 5). During the investigations the PC computer with Matlab-Simulink software is used. For virtual reality presentation, a system based on VRLM environment is used.

There are two basic and dominant control methods, which may be applied when haptic joystick is used: force-command and motion command (Harward, 2006; Zhuang and Canny, 2000). When the operator's joystick motion is measured and taken as an input and the device force is fed back to the user, impedance control is applied. When the force exerted by user on a joystick arm is measured and time varying position is fed back to the operator, the method is called admittance control.

The main aim of this study is to choose an optimal control algorithm, which then will be implemented in the control system of the real electrohydraulic drive with a haptic joystick. The first investigated algorithm was admittance control, which block scheme is shown in Fig. 6 . In this algorithm the drive position $y$ is controlled by applied electronics and operator feels the force $F_{j}$ which is proportional to the force occurring between the drive and the obstacle. In order to calculate this force two pressure sensors are installed in the cylinder chambers. 


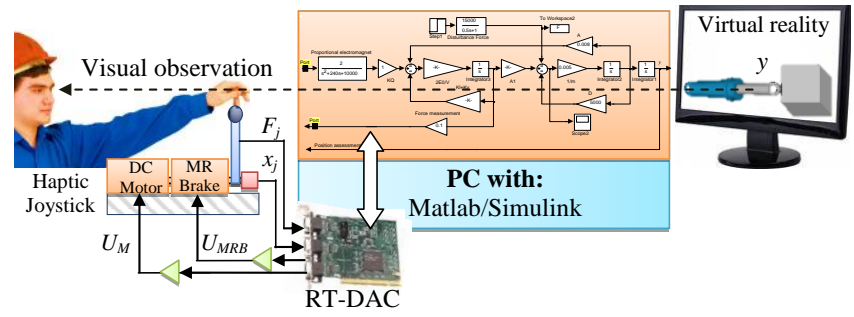

Fig. 5. The block scheme of investigation stand with real joystick

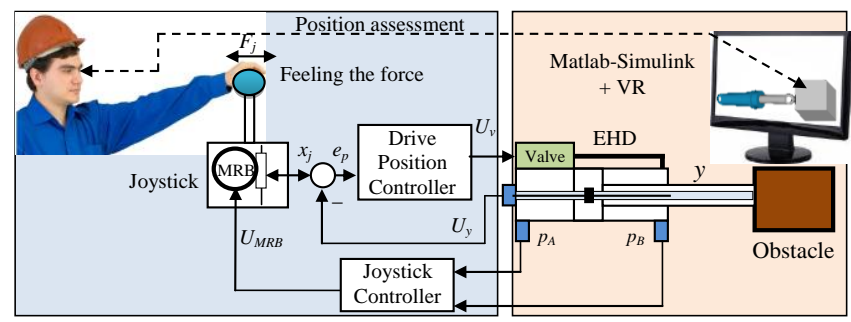

Fig. 6. Block diagram of admittance control

In the algorithm this force was calculated as pressure difference in the cylinder chambers, multiplied by controller gain coefficient. The force signal was sent as MR brake control voltage UMRF to the joystick controller.

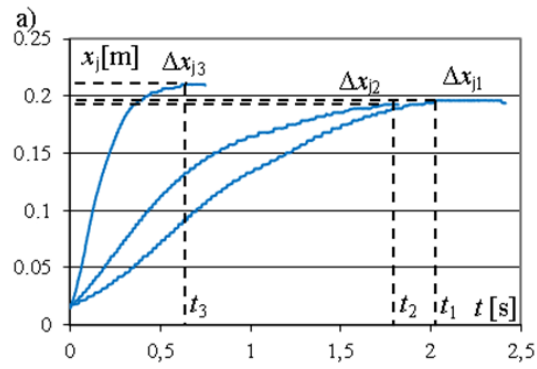

b)

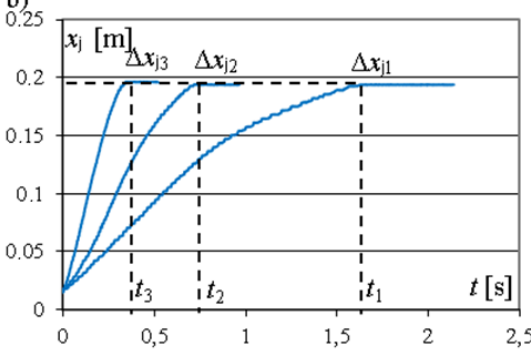

Fig. 7. The results obtained in simulation investigations of the admittance control system: a) without the feedback;

b) with the force feedback

During the investigations in which simulation model and VR visualization was used, the precision of electrohydraulic drive rod positioning in touching an obstacle, depending on the speed of movement, was investigated. Virtual obstacle was placed in a distance of $0.193 \mathrm{~m}$ from the initial position of the piston rod. In the investigations 15 operators tried to touch smoothly the obstacle with the hydraulic cylinder piston rod. The position of the joystick arm $x_{j}$ was measured and recorded during all the attempts made by operators. The best representative results obtained by a chosen operator are presented in Fig. 7. When the force feedback is disconnected (Fig. 7a), the operator is able to touch the obstacle in time $t_{1}=2.05 \mathrm{~s}$. In this case, the piston speed is about 0.1 $\mathrm{m} / \mathrm{s}$. If the hydraulic piston speed is about $0.2 \mathrm{~m} / \mathrm{s}$, the obstacle contact time is reduced to $t_{2}=1.8 \mathrm{~s}$, but position error is bigger i.e. equal to $\Delta x_{j 2}=6 \mathrm{~mm}$. At a piston speed equal to $0.5 \mathrm{~m} / \mathrm{s}$, the operator has stopped moving the joystick arm until about $t_{3}=0.6$ $\mathrm{s}$, but in this case the position error still is the largest, and reached $19 \mathrm{~mm}$. In practice, this means that the piston hit the obstacle hard. The obtained parameters are significantly improved when force feedback is switched on, as shown in Fig. 7b.

In the investigations made by different operators, the position error is not bigger than $3 \mathrm{~mm}$, which occurred only for the highest speed of the movement equal to $0.8 \mathrm{~m} / \mathrm{s}$. The shortest time, in which the operator is able to touch smoothly the obstacle by the piston rod was $t_{3}=0.32 \mathrm{~s}$.

In Fig. 8 the scheme block diagram of impedance control is presented. In this case, the force controller is used in the drive and the operator has used his visual observation of the drive in order to generate assumed force. The force feedback is assured by pressure sensors located in cylinder chambers.

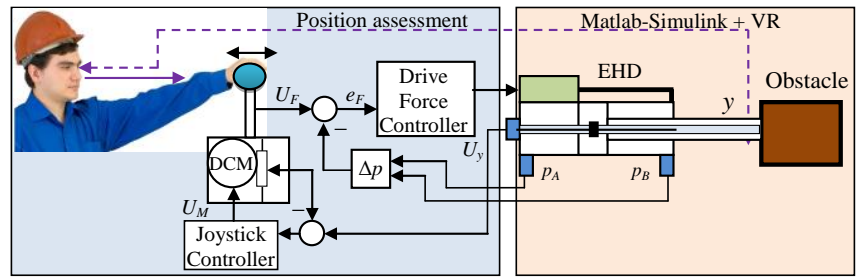

Fig. 8. Block diagram of modified impedance control algorithm
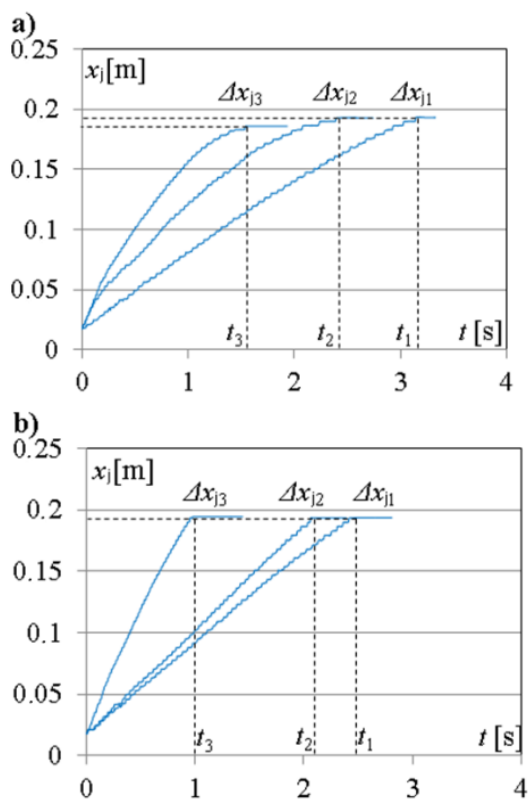

Fig. 9. The results obtained in simulation investigations of the modified impedance control algorithm a) without the feedback; b) with the force feedback

However, in order to use the haptic joystick for control of a drive with internal force feedback loop, additional solution should be used. Instead of the MR brake, the DC motor (DCM) needs to be installed in the joystick, which will set the joystick arm in a position corresponding to the current position of cylinder piston. The force on a joystick arm, measured by a strain gauge is used as an assumed signal $U_{F}$ for the internal force feedback loop. The joystick arm position signal $x_{j}$ is compared with the drive current position signal. 
The difference between these two signals is used by Joystick Controller for generation of the voltage signal to DC motor installed in a joystick. So, the joystick in the impedance control system can be categorized as active. In this way in the impedance control, the human operator feels in a joystick a current drive position, and sets the required force. The DC motor tries to set the joystick arm in a position which is proportional to the piston position. In Fig. 9. the simulation results are presented, which are obtained using the described above modified impedance control.

As it was in the previous case, the results show the improvement in positioning accuracy after the introduction of the haptic feedback. The highest position error occurred at the highest piston speed.

\section{INVESTIGATIONS OF THE ADMITTANCE AND IMPEDANCE CONTROL WITH THE USE OF HAPTIC JOYSTICK}

In Fig. 10 the block scheme of a test stand for testing of real electrohydraulic drive controlled by the haptic joystick, is shown. It consists of three main elements: the PC which acts as a control unit, a haptic joystick with its controller and electrohydraulic drive with a load cylinder and measurement equipment.

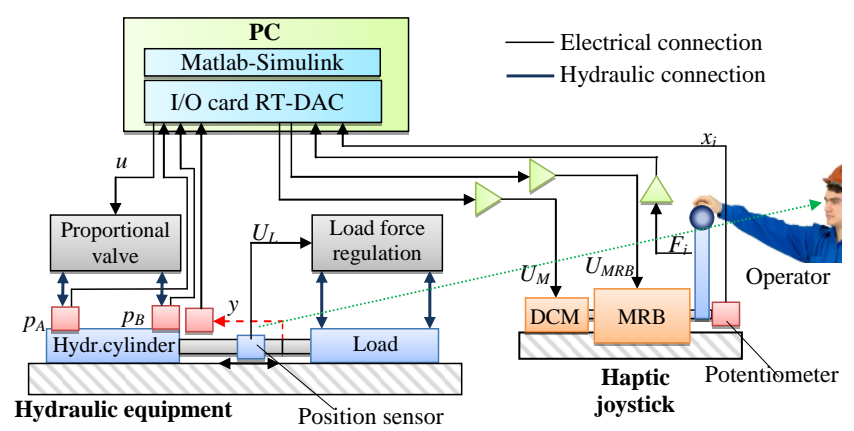

Fig. 10. The block diagram of research station for laboratory research

On the PC the program Matlab/Simulink is installed on which the implementation of control algorithm for hydraulic drive and haptic joystick is made. The controller inputs are: joystick arm position $x_{j}$ and joystick arm force $F_{j}$. Basing on them, the proportional valve voltage control signal $u$ is calculated and sent to the drive control card. In both hydraulic drive cylinder chambers the pressure sensors type MBS 1250 Danfoss with measurement range $0-25 \mathrm{MPa}(0-250 \mathrm{bar})$ and analog output $0-5 \mathrm{~V}$ are installed and connected by RT-DAC card in the control computer. The force generated by the piston rod of hydraulic cylinder is calculated as a difference of pressures in a cylinder chamber. This pressure difference signal is also used as a feedback signal, for calculation of the magnetorheological brake voltage control signal $U_{\text {MRB. }}$. The position of hydraulic cylinder piston is measured and additionally used for control of the proportional valve and DC motor $\left(U_{M}\right.$ voltage) installed in a joystick. The photo of the test stand is shown in Fig. 11.

The position of hydraulic cylinder piston is measured by the linkage sensor. Its measurement distance is equal to $1250 \mathrm{~mm}$ and analog output is in a range of $0-10 \mathrm{~V}$. For this sensor maximum measurement parameters are: speed $10 \mathrm{~m} / \mathrm{s}$; acceleration $300 \mathrm{~m} / \mathrm{s}^{2}$. The measured position nonlinearity error is $0.1 \%$.

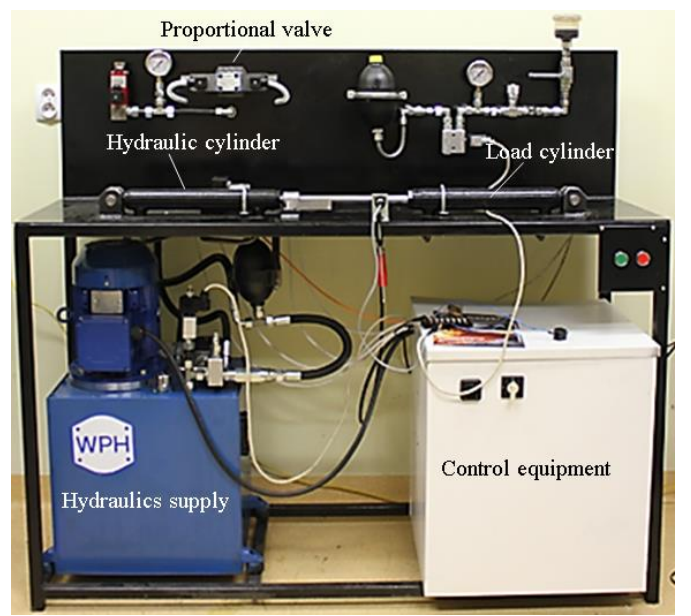

Fig. 11. The photo of hydraulic research station
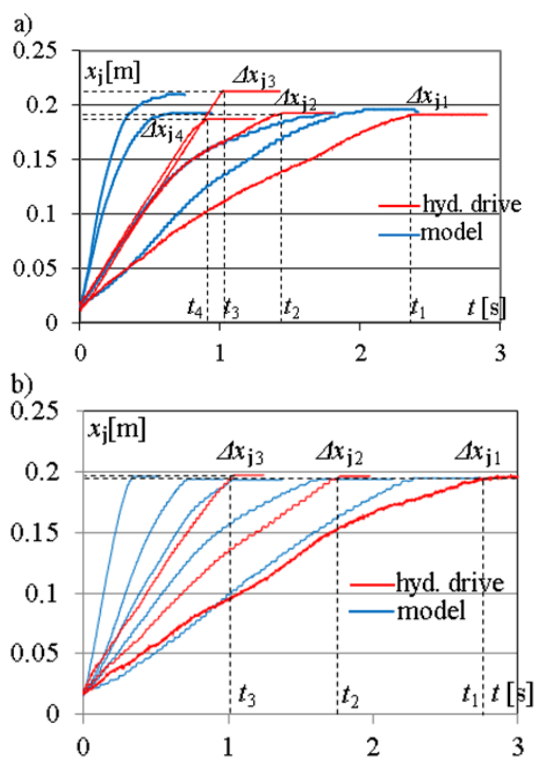

Fig. 12. Investigation results of control of electrohydraulic drive when admitance control was used: a) without the feedback; b) with the force feedback

In the empirical investigations 15 operators were involved, each of which has performed 3 tasks making 5 attempts for each task. The obstacle was located in this case $0.16 \mathrm{~m}$ from the initial position of the hydraulic cylinder piston rod. In Fig. 12, the best representation (obtained for one chosen operator) of all research with the use of admittance control, are presented.

In Fig. 12 the results obtained without and with admittance control are compared. In this figure curves obtained in simulation (red color) and in laboratory experiments are presented (blue color). In case when the haptic feedback is not active (Fig. 12a), during first attempt, when the velocity was about $0.8 \mathrm{~m} / \mathrm{s}$, the operator was able to touch the obstacle with an error of $\Delta x_{j 1}=2$ $\mathrm{mm}$ in a time $t_{1}=2.34 \mathrm{~s}$. In the second attempt, the velocity has increased to about $1.4 \mathrm{~m} / \mathrm{s}$ and the time was reduced to $t_{2}=1.4 \mathrm{~s}$, without changing the positioning error. In next two attempts the velocity was about $1.8 \mathrm{~m} / \mathrm{s}$ and the time was reduced to $t_{3}=1.05 \mathrm{~s}$ and $t_{4}=0.92 \mathrm{~s}$. However in these two cases the positioning error was $\Delta x_{j 3}=-2 \mathrm{~mm}$ and $\Delta x_{j 4}=20 \mathrm{~mm}$. The characteristics obtained when the force feedback is activated are shown in Fig. 12b. It is clearly visible that for all recorded curves the positioning errors are significantly reduced. For the biggest velocity equal to about 
$0.8 \mathrm{~m} / \mathrm{s}$ it is not higher than $4 \mathrm{~mm}$. Also the positioning time was reduced and the best result was $t_{3}=1 \mathrm{~s}$ which was $0.4 \mathrm{~s}$ faster than the time obtained when the force feedback was switched off. The comparison of curves obtained in simulation with curves obtained in real experiments have indicated close similarities of the run.

In Fig. 13 the results obtained when impedance method was applied are presented. If the haptic feedback is not activated the operator was able to touch the obstacle in an average time $t_{1}=4.07 \mathrm{~s}$ with velocity $0.04 \mathrm{~m} / \mathrm{s}$ and with positioning error equal to about $1 \mathrm{~mm}$. When the velocity was increased to $0.07 \mathrm{~m} / \mathrm{s}$ the positioning time was $t_{2}=3.15 \mathrm{~s}$. For the velocity $0.2 \mathrm{~m} / \mathrm{s}$ this time was reduced to $t_{3}=1.63 \mathrm{~s}$, but the positioning error was $\Delta x_{d 3}=-2$ $\mathrm{mm}$. The results obtained when the haptic feedback was on, are shown in Fig. 13b.
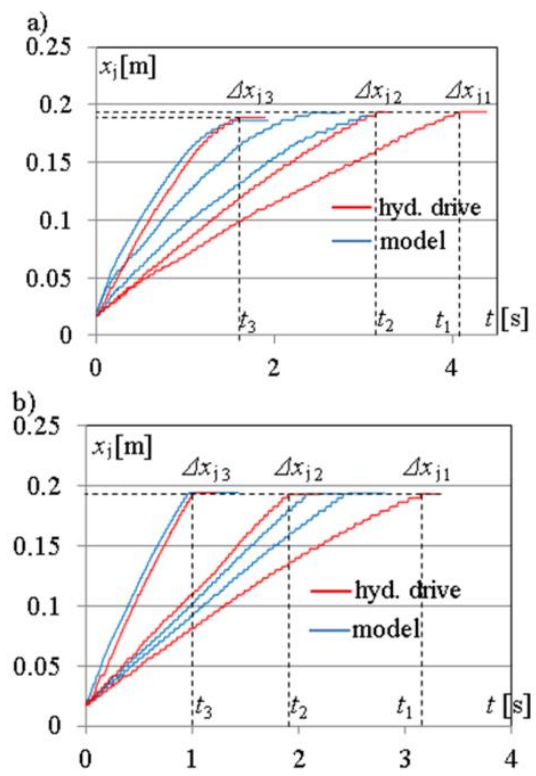

Fig. 13. Investigation results of control of electrohydraulic drive when impedance control was used: a) without the feedback; b) with the force feedback

In all attempts the positioning error was not bigger than $\Delta x_{d 3}=$ $1 \mathrm{~mm}$, and the shortest positioning time was about $t_{3}=1 \mathrm{~s}$, which was of about $2.15 \mathrm{~s}$ faster in comparison to results obtained without a feedback. Similarly to the results obtained if admittance control is used, also in impedance control the results have been significantly improved.

\section{CONCLUSION}

The paper presents the results of simulation and laboratory investigations of electrohydraulic drive control made by a human operator, who used a haptic joystick. In the presented work two main control methods are considered: admittance and impedance. The theoretical control model of these two methods are described and their simulation model is proposed. The simulation investigations are focused on checking how quickly and accurately can the operator control the electrohydraulic drive during touching the obstacle by using a one-axis joystick. The obtained simulation results enable to design and build control strategies for system composed of a haptic joystick and a real electrohydraulic drive.
Finally the whole system was built, implemented and investigated in laboratory environment. The empirical investigations have shown that the use of haptic joystick improved the accuracy of touching obstacles. The biggest position errors occurred when the operator controlled the hydraulic drive without any direct feedback and had limited opportunity to observe the moving object. This situation can occur in practice, e.g. while excavator works in a deep trench and where the excavator bucket interferes with a heavy object (stone, pipe). The smallest error was recorded when the drive was controlled when the haptic feedback was switched on. Fifteen operators have participated in the simulation and experimental investigations.

The results have shown that haptic control can improve the human feeling of forces between electrohydraulic drive and an obstacle and thanks to this, the manual control can be more accurate and safer.

\section{REFERENCES}

1. Adams R.J., Hannaford B. (1999), Stable haptic interaction with virtual environments, IEEE Transactions on Robotics and Automation, 15(3), 465-474.

2. Frankel J.G. (2004), Development of a Haptic Backhoe Testbed, MS thesis, The Georgia Institute of Technology, G.W. Woodruff School of Mechanical Engineering.

3. Harward V. (2006), Haptic synthesis, Proc. 8th International IFAC Symposium on Robot Control, SYROCO, 19-24.

4. Kontz M.E. (2002), Haptic Enhancement of Operator Capabilities in Hydraulic Equipment, Masters of Science Thesis, School of Mechanical Engineering, Georgia Institute of Technology.

5. Kontz M.E. (2007), Haptic control of hydraulic machinery using proportional valves, Doctorate thesis, G.W. Woodruff School of Mechanical Engineering.

6. Kudomi S., Yamada H., Muto T. (2000), Development of a hydraulic master-mlave mystem for telerobotics, Proceedings of 1st FPNIPhD Symposium, Hamburg, 467-474.

7. Li P.Y. (2000), Towards safe and human friendly hydraulics: the passive palve, ASME Journal of Dynamic Systems, Measurement and Control, 122(3), 402-409.

8. Milecki A., Myszkowski A., (2009), Modelling of electrohydraulic servo drive used in very low velocity applications, International Journal of Modelling, Identification and Control, 7(3), 246-254.

9. Oh K.W., Kim D., Hong D., Park J.-H., Hong S. (2008), Design of a haptic device for excavator equipped with crusher, Book the 25th International Symposium on Automation and Robotics in Construction, ISARC-2008, Publisher Vilnius Gediminas Technical University Publishing House "Technika", 202-208.

10. Park H.-J., Lee S., Kang S.-K., Kang M.-S., Min-Su, Han S.Ch. (2011) Experimental study on hydraulic signal compensation for the application of a haptic interface to a tele-operated excavator, Proceedings of the 28th ISARC, Seoul, Korea, 298-303.

11. Won Oh K., Kim D., Kim N., H., Hong D. (2011), The virtual environment for force-feedback experiment of excavator using a novel designed haptic device, Proceedings of the 28th ISARC, Seoul, Korea, 51-56.

12. Zarei-nia K., Goharrizi A. Y., Sepehri N., Fung Wai-keung (2009), Experimental evaluation of bilateral control schemes applied to hydraulic actuators: a comparative study, Transactions of the Canadian Society for Mechanical Engineering, 33, 377-398.

13. Zee van der L.F. (2009), Design of a haptic controller for excavators, Thesis MScEng, Electrical and Electronic Engineering, University of Stellenbosch.

14. Zhuang, Y., Canny, J. (2000), Haptic interaction with global deformations, Proc. IEEE Robotics and Automation Conference, 2428-2433. 